\title{
Microstructural Path Analysis of Martensite Burst
}

\author{
Paulo Rangel Rios ${ }^{\mathrm{a}, \mathrm{b}, *}$, José Roberto Costa Guimarães ${ }^{\mathrm{a}, \mathrm{c}}$ \\ ${ }^{a}$ Escola de Engenharia Industrial Metalúrgica de Volta Redonda, \\ Universidade Federal Fluminense - UFF, \\ Av. dos Trabalhadores, 420, CEP 27255-125 Volta Redonda - RJ, Brazil \\ ${ }^{\mathrm{b}}$ RWTH Aachen University, Institut für Metallkunde und Metallphysik, D-52056 Aachen, Germany \\ 'Mal. Moura 338H/22C, CEP 05641-000 São Paulo - SP, Brazil
}

Received: November 9, 2009; Revised: February 10, 2010

\begin{abstract}
Modeling the martensite reaction requires reckoning with spatial aspects of the reaction. For that, we used formal kinetics, more specifically, the microstructural path method (MPM) to analyze the microstructure observed in a burst. The microstructural path analysis revealed that the size of the spread cluster in extended space, characterized by the Vandermeer and Juul-Jensen's impingement compensated mean intercept length, $\lambda_{G}$, remained constant, independently of the parent austenite grain size. Moreover, current analysis introduced a purely formal description of the reaction progress by taking the parent austenite grain size as the progress variable. This description worked very well and resulted in a relationship between the volume fraction of partially transformed austenite, $V_{V G}$, and austenite grain size, $\lambda_{G}$. The significance of these findings in the light of the advantages and disadvantages of formal kinetics is discussed.
\end{abstract}

Keywords: martensitic phase transformation, kinetics, nucleation, microstructure, analytical methods

\section{Introduction}

Martensite is a displacive phase transformation in which transformation shear strains are significantly higher than the transformation homogenous strains. This characteristic prompts microstructural heterogeneity, influences plate's shape, and can lead to a diversity in kinetics with a corresponding diversity of spatial arrangement of the transformation product. Since a martensite unit quickly reaches its final size, further transformation requires additional nucleation events that are, frequently, correlated with previous ones. In other words, martensite is a nucleation controlled reaction with a significant autocatalytic component.

One of the unique and intriguing features that athermal martensite presents in some alloys is the so called "martensite burst" or simply "burst". As soon as the alloy reaches the martensite start temperature, $M_{s}$, the burst may even be triggered, even by a single nucleation event $^{1}$, resulting in a substantial volume fraction of martensite, up to $80 \mathrm{vol} .(\%)$ in some cases. The size of the burst depends on the driving force (burst temperature), but the size of the plates formed is that is controlled by the austenite grain size and by the arrangement of the plates. The propagation of martensite occurs rather quickly, so that in a burst spread and fill-in are completed almost instantaneously. An audible sound may accompany the burst.

Concerning spatial distribution of transformed regions, it is important to recall that the martensite reaction does not start simultaneously in all austenite grains as the experiment of Cech and Turnbull ${ }^{2}$ with particulate Fe-30 wt.(\%) Ni demonstrates. The probability that a particle transforms depends upon the propagation of nucleation at the reaction temperature. The fraction of particles transformed at a temperature $T$ has been described by Cohen and Olson ${ }^{3}$ (Equation 1).

$$
f=1-\exp \left(-q \cdot n_{V}\right)
$$

where $q$ is the particle volume and $n_{V}$ the density of nucleation sites. On the other hand, the fill-in of these particles with the martensite units is an autocatalytic process. The volume fraction of martensite in the particles is determined by the quenching temperature or driving force ${ }^{2}$.

In the case of bulk material, the connectivity of the grains allows the transformation in one grain to induce the propagation of a nucleation site in an untransformed neighbor, resulting in a cluster of partially transformed grains. This cluster of partially transformed grains is normally designated as a "spread event" or 'spread-cluster'. The collection of these single spread events can be defined as the 'spread' or 'martensite spread'. This process is restarted by another pioneer nucleation elsewhere as well as by further transformation within the partially transformed grains, in what is normally called the 'fill-in'.

Thus, for convenience, we describe a spread event comprising three steps: 1) The prime nucleation event by propagation of a preexistent nucleation site; 2) The autocatalytic fill-in of the grain with martensite units, and 3) The impingement of some of these plates onto the GB. The latter step causes the spreading of the reaction from the grain where the prime event occurs into a neighbor and so on until exhaustion. Let the average number of martensite per grain be $q N_{V}$. Therefore, the analog of Equation 1 applicable to the burst transformation in bulk polycrystal is (Equation 2):

$$
V_{V G}=1-\exp \left(-P_{i} q N_{V}\right)
$$

where $P_{I}$ is the probability that a martensite unit impinges on the GB. This expression of $V_{V G}$ has been validated before ${ }^{5-7}$, and $P_{i} \approx 0.1$ in the case of lenticular martensite forming in Fe-31 mass $\% \mathrm{Ni}-0.02$ mass $\% \mathrm{C}$.

Figure 1 shows clusters of partially transformed grains formed in bursting $\mathrm{Fe}-31 \% \mathrm{Ni}-0.02 \% \mathrm{C}$ cooled to the martensite start temperature that coincides with the burst temperature, $M_{B}$. It is conspicuous that grain boundaries are impervious to martensite propagation and 


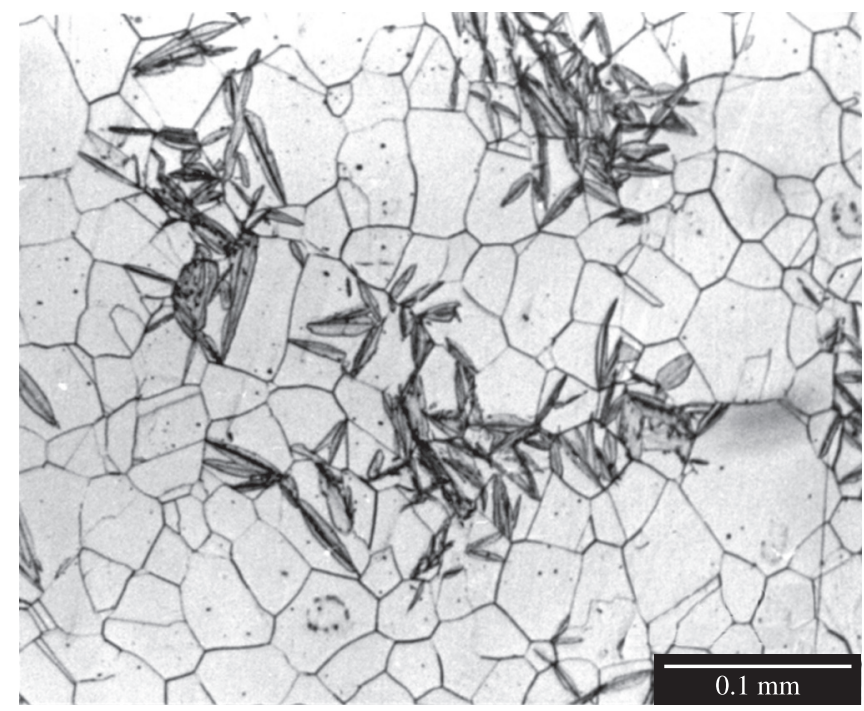

(a)

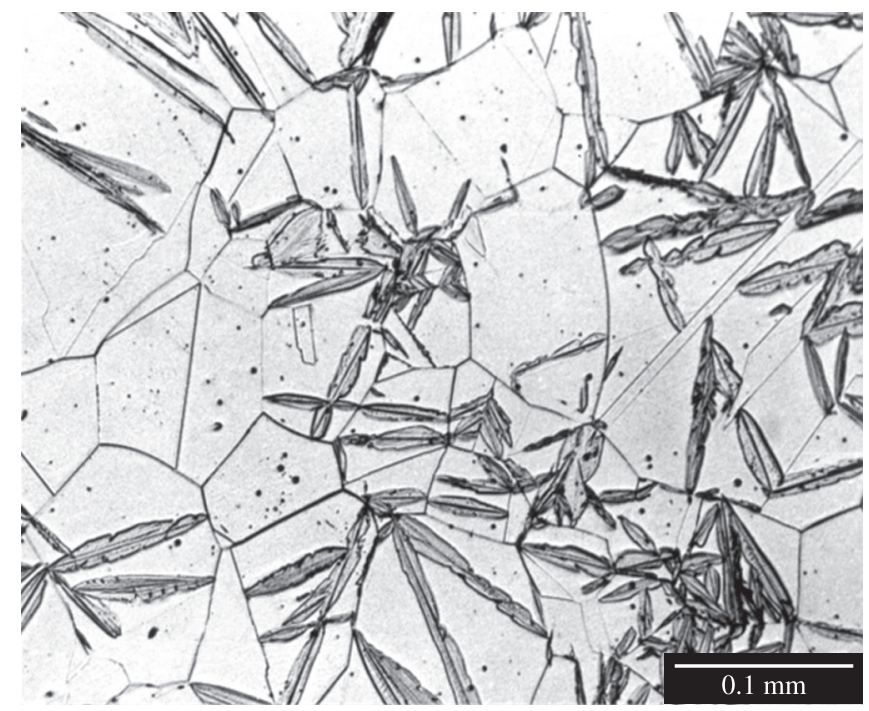

(b)

Figure 1. Optical photomicrographs of a Fe-31\%Ni-0.02\% C cooled to the burst temperature, 212-222 K: a) Fine grain size, b) Coarse grain size. The reaction is better spread over the material with the coarse grain size.

blocks the propagation of the midrib, the reaction's fast leading step. The sudden stoppage of a midrib at a grain boundary generates a peak perturbation that can interfere with the transfer of transformation strains to the matrix as well as with subsequent transformation events $^{8-11}$. The martensite units forming zigzags are observed in every grain where the transformation occurred. The zigzags result from the coupling of the transformation shape strain, a form of autocatalysis that helps to optimize the discharge of transformation strain ${ }^{12-14}$. In bulk polycrystalline material we expect grain boundaries to provide a suitable environment for the initial nucleation of martensite ${ }^{10,14-18}$. Hence, the impingement of a martensite unit onto a grain boundary should provide stimulus for the propagation of a nucleation site in the next grain. It is notable that the balance of these two effects results in the spread of the reaction. It is a contention that in absence of martensite units impinging onto grain boundaries, a burst would spread sluggishly as in isothermal martensite.

These experimental facts indicate that modeling martensite burst requires reckoning with the spatial aspects of the reaction. For this purpose, formal kinetic modeling and the microstructural path method, briefly described in the following, have shown to be useful tools ${ }^{19-23}$.

The basis of formal kinetic modeling is the early work of Johnson-Mehl ${ }^{24}$, Avrami ${ }^{25}$ and Kolmogorov ${ }^{26}$ (JMAK), which used only a single microstructural descriptor, $V_{V}$ JMAK's work was subsequently extended by DeHoff and Gokhale ${ }^{27,28}$, who proposed the use of an additional microstructural descriptor, $S_{V}$, and the associated concept of microstructural path. Vandermeer and coworkers ${ }^{29}$ extended the microstructural path concept and crystallized it in an all round theoretical treatment covering variable nucleation and growth rates as well as non-spherical regions: the microstructural path method (MPM) or microstructural path analysis (MPA). Recently, Rios and Villa ${ }^{30,31}$ have extended previous work using stochastic geometry methods.

The microstructural path method was originally developed to deal with recrystallization and later applied also to diffusional transformations and grain growth but it is in fact quite general and has been used to model a diversity of heterogeneous transformations in both metallic and non-metallic materials.
Table 1. Bulk Fe-31 mass\% Ni-0.02 mass\% C burst parameters ${ }^{5-6}$.

\begin{tabular}{ccccccc}
\hline$M_{B}(\mathrm{~K})$ & $S_{V \gamma}\left(\mathrm{mm}^{-1}\right)$ & $\lambda_{\gamma}(\mu \mathrm{m})$ & $V_{V}$ & $V_{V G}$ & $V_{V} / V_{V G}$ & $S_{V G}\left(\mathrm{~mm}^{-1}\right)$ \\
\hline 212 & 76.5 & 26 & 0.01 & 0.07 & 0.14 & 1.04 \\
214 & 71.7 & 28 & 0.05 & 0.14 & 0.36 & 1.82 \\
220 & 40.8 & 49 & 0.05 & 0.23 & 0.22 & 2.8 \\
222 & 31.3 & 64 & 0.18 & 0.64 & 0.28 & 5.77 \\
220 & 14.1 & 142 & 0.23 & 0.84 & 0.27 & 3.93 \\
\hline
\end{tabular}

Recently, Rios and Guimarães have successfully used MPM to analyze martensite spread of both athermal ${ }^{19}$ and isothermal martensite $^{20}$. In this work the same MPM methodology is applied to the martensite burst.

\section{Experimental Description of the Burst in a Fe-31 mass\% Ni-0.02 mass\% C Alloy}

The overall spread of martensite transformation has been conveniently described by the volume fraction of clusters of partially transformed grains, $V_{V G}$, and by the area per unit volume of clusters' boundaries, $S_{V G}{ }^{5}$. These two stereological parameters, obtainable by point and lineal counting on a planar surface, have been used in formal analyses and in computer-modeling the martensite spread ${ }^{6}$. $V_{V G}$ increases with the volume fraction transformed and readily responds to a variation in the austenite grain size, whereas $S_{V G}$ goes through a maximum at an intermediate value of $V_{V G}{ }^{5}$. In this work we analyze published quantitative metallographic data from the burst in a Fe-31 mass \% Ni-0.02 mass\% C alloy with different grain sizes ${ }^{4-7}$. Table 1 provides a quantitative description of the microstructure of the initial burst in bulk $\mathrm{Fe}-31 \% \mathrm{Ni}-0.02 \% \mathrm{C}$. It shows the aforementioned quantities $V_{V G}$, and $S_{V G}$ as well as: austenite grain size, $\lambda$, and area per unit of volume, $S_{V \gamma}$, and martensite volume fraction, $V_{V}$. The burst temperatures, $M_{B}$, are also included. The data in Table 1 were compiled from the original publications ${ }^{5-6}$. Non-conspicuous data points were ignored. Small variations from the compilation were averaged out by reiteration. Originally, the compiled data did not bear error bars. However the quantitative stereological methods used by the original sources suggest that it is not unreasonable ascribing a relative error 
of $\pm 10 \%$, although errors could be larger in the case of parameters derived from the ratio of two measured quantities.

It can be seen in Table 1 that the range of burst temperatures, $M_{B}$, is narrow $(212-222 \mathrm{~K}$ ). However, the mean intercept length, $\lambda$, ( or the area per unit volume of grain boundaries, $S_{V \gamma}$ ) exhibit a significant variation. It is noticeable that $V_{V G}$ varies significantly with $S_{V \gamma}$, compared with the volume fraction of martensite in the clusters given by $V_{V} / V_{V G}$, likewise observed in particulate $\mathrm{Fe}-30 \% \mathrm{Ni}$ material ${ }^{5}$.

\section{Microstructure Path Method Applied to the Martensite Burst}

The classical expressions for the clusters of partially transformed grains located in space according to a homogeneous Poisson point process $^{30,31}$ are well-known: Equations 3, 4.

$$
\begin{gathered}
V_{V G}=1-\exp \left(-V_{V G}^{E}\right) \\
S_{V G}=S_{V G}^{E}\left(1-V_{V G}\right)
\end{gathered}
$$

where $V_{V G}^{E}$ and $S_{V G}^{E}$ are extended quantities, i. e. expressed without considering impingement.

Furthermore, weighing the concepts $\operatorname{in}^{32-34}$, we use the impingement compensated cluster's mean intercept, $\lambda_{G}^{E}$, to describe the cluster of partially transformed grains (Equation 5),

$$
\lambda_{G}^{E}=\frac{4 V_{V G}^{E}}{S_{V G}^{E}}
$$

Combining the equations we obtain a relationship between $S_{V C}$ and $V_{V G}$, the microstructural path: Equation 6 .

$$
\frac{S_{V G}}{\left(1-V_{V G}\right)}=\frac{4}{\lambda_{G}^{E}} \ln \left(\frac{1}{1-V_{V G}}\right)
$$

where $\lambda_{G}^{E}$ has the same units as $\left(\mathrm{S}_{V G}\right)^{-1}$.

Figure 2 shows that the plot of the microstructural path is an almost perfect straight line, the correlation coefficient is $R^{2}=0.99$ with $\lambda_{G}^{E}=287 \mu \mathrm{m}$. It is interesting to point out that the mean intercept length, $\lambda_{G}$, for a "real" cluster is $\lambda_{G}=4 V_{V G} / S_{V G}$. For small volume

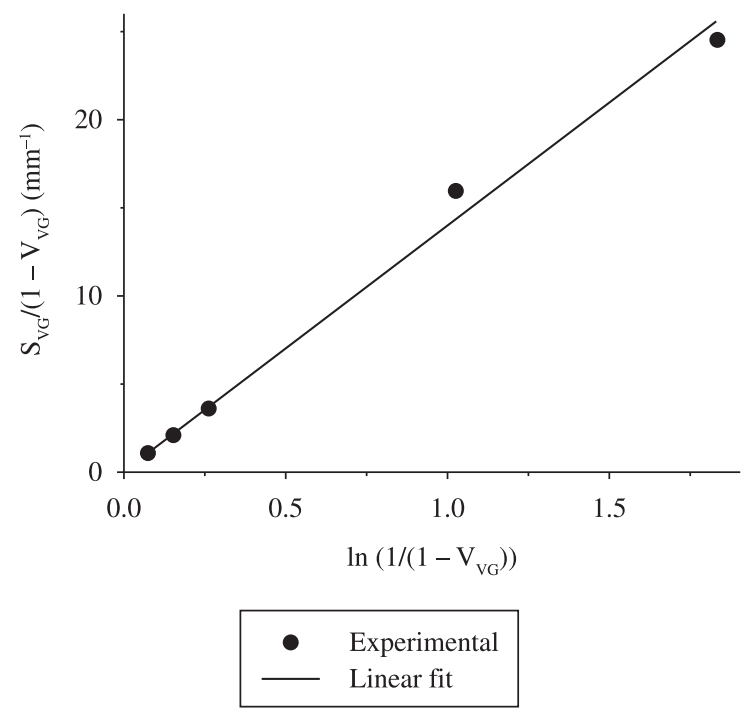

Figure 2. Plot of Equation 6 with experimental data on martensite burst, see Table 1, on a Fe-31 mass\% Ni-0.02 mass\% C alloy. fractions, $\mathrm{V}_{\mathrm{vG}}<<1, \lambda_{G}^{E}$ approaches $\lambda_{G}$ because the impingement effect is small. This can be easily checked noticing that $V_{V G}=0.07$ and $S_{V G}=1.04 \mathrm{~mm}^{-1}$ (Table 1) result in $\lambda_{G}=269 \mu \mathrm{m}$, which is comparable to $\lambda_{G}^{E}=287 \mu \mathrm{m}$.

In previous work ${ }^{19,20}$, also using MPM, the present authors have described the spread event as a cluster of tetrakaidecahedron grains. Clearly, one model can be easily converted into another, if necessary. It can be shown that the average number, $\gamma$, of grains comprising such a cluster in extended volume is related to $\lambda_{G}^{E}$ by $\lambda_{G}^{E}=\gamma^{1 / 3} \lambda_{\gamma}$.

It is worthy of note that the progress of athermal martensitic spread could be formally described by assuming that mean number of grains of the spread-cluster in extended space remained constant throughout the reaction, $\gamma \approx 68$. Moreover, this number of partially transformed austenite grains constituting a spread-cluster did not change with grain size. Since $\gamma$ was independent of the austenite grain size, the mean size of the spread cluster in extended space was larger for the coarser grained austenite.

The influence of the austenite grain size on the behavior of the burst is very different from the behavior of the athermal spread just described. In the burst the size of the spread-cluster is independent of the austenite grain size. The material with coarse grain size has fewer grains per cluster, that is, $\gamma$ decreases with increasing the austenite grain size at nearly constant (burst) temperature. For a spherical cluster containing tetrakaidecahedral grains the number of grains located within the cluster, $\gamma_{S}$, is ${ }^{19,10}$

$$
\gamma_{S}=\frac{243 \pi}{1024}\left(\frac{\lambda_{G}^{E}}{\lambda_{\gamma}}\right)^{3}
$$

For a tetrakaidecahedral cluster containing tetrakaidecahedron grains the number of grains located within the cluster, $\gamma$, is ${ }^{20}$ :

$$
\gamma \cong 0.74 \gamma_{S}
$$

Equations 7 and 8 imply that a significant number of grains, roughly 900 , are involved in the spread-cluster when the grain size is small, $26-28 \mu \mathrm{m}$. This number significantly decreases to about 200 grains when the grain size is about $49 \mu \mathrm{m}$. The figures indicated that the number of parent austenite grains contained in a spreadevent in a burst may be significantly larger than the number of parent austenite grains in a spread event induced by further cooling the alloy below the burst temperature.

\section{Formal Analysis of Spread Kinetics Taking the Austenite Grain Size as the Progress of Reaction Variable}

Formal analysis using the classical JMAK approach implies that evolution of the transformed region is related to nucleation and growth. In the case of the martensite spread, the transformed regions, that is, the spread-clusters, quickly reach their final size because the reaction is largely nucleation controlled and growth is very fast. Further martensite nucleation in athermal martensitic transformation requires further cooling, so that 'rate' is a 'temperature rate' that was found constant ${ }^{19}$.

In the realm of the MPM, the burst is a nearly instantaneous event, so that the observed microstructure provides only one reaction point along its microstructural path. Nevertheless, by utilizing alloys with different grain sizes other reaction points along their respective microstructural paths can be found, provided that the martensite units maintain their morphology. These points can be described by a common $S_{V G}^{E}$ vs. $V_{V G}^{E}$ curve, as shown in Figure 2, if $\lambda_{G}^{E}$ remains approximately constant with grain size. This is what has been found here: in extended space the cluster-spread size during martensite 
burst is roughly independent of the austenite grain size. Bearing this in mind one may write (Equations 9, 10).

$$
V_{V G}^{E}=\frac{1}{4} S_{V G}^{E} \lambda{ }_{G}^{E}
$$

and

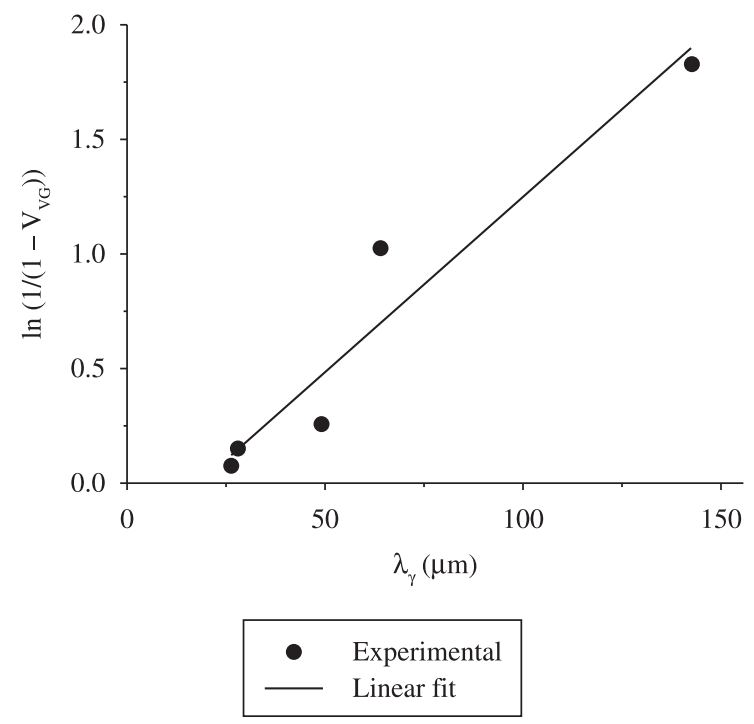

Figure 3. Plot of Equation 14 with experimental data on martensite burst, see Table 1, on a Fe-31 mass\% Ni-0.02 mass\% C alloy. Fitted parameters are given in Table 2 .

Table 2. Bulk Fe-31 mass $\%$ Ni- 0.02 mass $\% \mathrm{C}$ burst parameters $-\lambda_{G}^{E}$, $\mathrm{I}_{\mathrm{B}}$ and $\lambda_{\gamma 0}$, obtained from the analysis of experimental data.

\begin{tabular}{cccccc}
\hline \multicolumn{2}{c}{ Equation 6 } & \multicolumn{4}{c}{ Equation 14 } \\
\hline$\lambda_{G}^{E}(\mu \mathrm{m})$ & $R^{2}$ & $\mathrm{~A}\left(\mu \mathrm{m}^{-1}\right)$ & $\lambda_{\gamma 0}(\mu \mathrm{m})$ & $\mathrm{I} B\left(\mathrm{~mm}^{-3} \mathrm{~mm}^{-1}\right)$ & $R^{2}$ \\
287 & 0.99 & 0.01526 & 18 & 365 & 0.91 \\
\hline
\end{tabular}

$$
S_{V G}^{E}=n_{V} s_{G}^{E}
$$

where $n_{V}$ is the number of spread-clusters per unit of volume and $S_{G}^{E}$ is the area of a spread-cluster, which also remains constant with grain size since the spread-cluster size $\lambda_{G}^{E}$ remains approximately constant with grain size.

For formal purposes, we can define a 'spread rate', $I_{B}$, with reference to the austenite grain size, assuming that temperature effects can be neglected owing to the narrow temperature range of the burst, 212-222 K. (Equations 11, 12, 13, 14).

A list of symbols used in this paper may be found in Table 3 .

$$
\mathrm{I}_{B}=\frac{d n_{V}}{d \lambda_{\gamma}}
$$

or

$$
n_{V}=\mathrm{I}_{B}\left(\lambda_{\gamma}-\lambda_{\gamma 0}\right)
$$

And from Equations 1, 6-7

$$
\begin{aligned}
& V_{V G}=1-\exp \left(-\frac{\lambda_{G}^{E} s_{G}^{E} \mathrm{I}_{B}}{4}\left(\lambda_{\gamma}-\lambda_{\gamma 0}\right)\right) \\
& \text { Finally, with } A=\frac{\lambda_{G}^{E} s_{G}^{E} \mathrm{I}_{B}}{4}
\end{aligned}
$$

$$
\ln \left(\frac{1}{1-V_{V G}}\right)=A\left(\lambda_{\gamma}-\lambda_{\gamma 0}\right)
$$

Figure 3 shows the plot of $\ln \left(\frac{1}{1-V_{V G}}\right)$ vs. $\lambda_{\gamma}$. It gives a straight line with a correlation coefficient is $R^{2}=0.91$. Thus, the equation above satisfactorily describes the fact that the volume fraction of the spread does depend on austenite grain size even though its size in extended space $\lambda_{G}^{E}$ is approximately independent of grain size.

Table 3. List of symbols used in this paper and their definitions.

\begin{tabular}{ll}
\hline Symbol & martensite start temperature; \\
\hline$M_{s}$ & martensite burst temperature; \\
$M_{B}$ & martensite volume fraction; \\
$V_{V}$ & volume fraction of material in grains partially transformed grains, that is, volume fraction of all grains in all clusters \\
$V_{V G}$ & of martensite spread; \\
$S_{V G}$ & area of interface per unit volume of material between a cluster of partially transformed grains and the untransformed \\
$V_{V G}^{E}, S_{V G}^{E}$ & parent matrix, that is, area of interface per unit volume the martensite spread; \\
$S_{V}^{E}$ & the subscript ext added to these quantities mean that they are extended quantities, that is, that they are calculated \\
$\lambda_{G}^{E}$ & ignoring impingement among growing regions. \\
$\gamma, \gamma_{S}$ & volume of a single cluster of austenite grains partially transformed to martensite comprising a number $\gamma$ of austenite \\
$N_{V \gamma}$ & the impingement compensated cluster's mean intercept length; \\
$q$ & number of grains in a cluster of partially transformed austenite grains that constitute a spread event; \\
$S_{V \gamma}$ & number of austenite grains per unit volume in the parent austenite matrix; \\
$\lambda_{\gamma}$ & grain boundary area per unit volume of parent austenite matrix grains; \\
$n_{V}$ & mean intercept length of parent austenite matrix intercept, $S_{V}^{m}=2 / \lambda ;$ \\
$\mathrm{I}_{B}$ & total number per unit volume of active nuclei at a certain grain size; \\
$A$ & spread nucleation rate, with reference to the austenite grain size;
\end{tabular}


A summary of fitted parameters of can be found in Table 2. For a grain size $\lambda_{\gamma 0}=18 \mu \mathrm{m}$ it is predicted that $V_{V G}=0$. This value of $\lambda_{\gamma 0}=18 \mu \mathrm{m}$ is remarkably close to the value of $5-15 \mu \mathrm{m}$ calculated from a previous work from one of the authors ${ }^{11}$.

\section{Discussion}

In previous papers by the same authors ${ }^{19,20}$, formal kinetics methodology was able to successfully describe athermal and isothermal martensite. Likewise, the same methodology could also successfully describe the geometric and kinetic characteristics of the martensite burst. It is important to stress that in all three cases, isothermal, athermal and burst, the methodology was rigorously applied.

The microstructural path analysis displayed in Figure 2 revealed that the size of the spread cluster in extended space, characterized by the impingement compensated cluster's mean intercept, $\lambda_{G}^{E}$, remained constant, independently of the parent austenite grain size. This in contrast with the results previously obtained for athermal and isothermal martensite in which the number of austenite grains in the spread-cluster remained constant but the size of the spread-cluster increased with parent austenite grain size.

Moreover, current analysis introduced a purely formal description of the reaction progress by taking the parent austenite grain size as the progress variable. This description worked very well and resulted in a relationship between the volume fraction of partially transformed austenite, $V_{V G}$, and austenite grain size, $\lambda_{\gamma}$.

However successful formal kinetics has been in modeling these situations it is important to highlight the limitations of the results obtained here. Formal kinetics is a general geometric/kinetic approach. It does not make any assumptions concerning the mechanisms or the causes whereby the relationships found here are established. In other words, the methodology reveals very well the general kinetic/ geometric relationships among relevant measurable stereological quantities. Yet, it cannot explain on its own the physical mechanisms causing these relationships to occur in the first place.

In order to investigate the physical causes behind the increase in $V_{V G}$ with $\lambda_{G}$ or why $\lambda_{G}^{E}$ remains constant during the burst it is necessary to carefully consider several factors pertaining to the martensite plate formation. These factors are, for example, austenite grain partition, induction of martensite transformation by a plate impinging on an austenite grain boundary, i. e. autocatalysis, among others. Thus, it is necessary considering the spatial aspects of the martensite plate formation, which would be a step further, beyond the scope of this paper.

In spite of the limitations discussed above, formal kinetics reveals important geometric/kinetic facts about the martensite burst that constitute a solid foundation upon which further understanding can be built upon.

\section{Summary and Conclusions}

In summary, the utilization of formal kinetics methodology to analyze the microstructure observed in a burst clarified the spatial aspects of the martensite burst.

The microstructural path analysis displayed in Figure 2 revealed that the size of the spread cluster in extended space, characterized by the impingement compensated cluster's mean intercept, $\lambda_{G}^{E}$, remained constant, independently of the parent austenite grain size.

Moreover, the analysis carried out in this work introduced a purely formal description of the reaction progress by taking the parent austenite grain size as the progress variable. This description worked very well and resulted in a relationship between the volume fraction of partially transformed austenite, $V_{V G}$, and austenite grain size, $\lambda_{\gamma}$.

\section{Acknowledgements}

P. R. Rios is grateful to Conselho Nacional de Desenvolvimento Científico e Tecnológico, CNPq, and to Fundação de Amparo à Pesquisa do Estado do Rio de Janeiro, FAPERJ, for financial support. P. R. Rios would also like to thank the Humboldt Foundation for the Humboldt Research Award and Professor Günter Gottstein for his hospitality during the author's stay at Institut für Metallkunde and Metallphysik of RWTH-Aachen where part of this work has been done.

\section{References}

1. Entwisle AR and Feeney JA. In: The Mechanism of phase transformations in crystalline solids. Nicholson RB (editor). London: Institute of Metals; 1969. p. 156-161.

2. Cech RE and Turnbull D. Heterogeneous nucleation of the martensite transformation. Transactions AIME. 1956; 206(2):124-132.

3. Cohen M and Olson GB. Martensitic nucleation and the role of the nucleation defect. Supplement Transactions Japan Institute of Metals. 1976; 17:93-98.

4. Guimarães JRC. Initial nucleation sites, autocatalysis and the spread of the martensite burst in Fe-31.9Ni-0.02C. Materials Science and Engineering. 1987; 95:217-224.

5. Guimarães JRC and Gomes JC. Metallographic study of influence of austenite grain-size on martensite kinetics in Fe-31.9 Ni-0.02C. Acta Metallurgica. 1978; 26(10):1591-1596.

6. Guimarães JRC and Saavedra A. A computer-assisted analysis of the spread of martensite-transformation. Materials Science and Engineering. 1984; 62(1):11-15.

7. Guimarães JRC. Athermal martensite: genesis of microstructure and transformation curves. Materials Science and Engineering A. 2008; 476(1-2)106-111.

8. Kajiwara S. Roles of dislocations and grain-boundaries in martensite nucleation. Metallurgical Transactions A 1986, 17A (10)1693-1702.

9. Hayzelden C and Cantor B. The martensite-transformation in Fe-Ni-C alloys. Acta Metallurgica. 1986; 34(2):233-242.

10. Takaki S, Fukunaga K, Syarif J and Tsuchiyama T. Effect of grain refinement on thermal stability of metastable austenitic steel. Materials Transactions. 2004; 45(7):2245-2251.

11. Guimarães JRC. Excess driving force to initiate martensite transformation in fine-grained austenite. Scripta Materialia. 2007; 57(3):237-239.

12. Machlin ES and Cohen M. Burst phenomenon in the martensitic transformation. Transactions AIME. 1951; 191(9):746-754.

13. Bokros JC and Parker ER. Mechanism of martensite burst transformation in Fe-Ni single crystals. Acta Metallurgica. 1963; 11(12):1291-1301.

14. Yang DZ, Sandvik BPJ and Wayman CM. On the substructure of athermal and isothermal martensites formed in an fe-21ni-4mn alloy. Metallurgical Transactions. 1984; 15A(8):1555-1562.

15. Zhang W, Jin YM and Khachaturyan AG. Phase field microelasticity modeling of heterogeneous nucleation and growth in martensitic alloys. Acta Materialia. 2007; 55(2): 565-574.

16. Tsukaki $\mathrm{K}$ and Maki T. The effect of cooling rate on the morphology of lath martensite in Fe-Ni alloys. Journal of the Japan Institute of Metals. 1981; 45(2):126-134.

17. Krauss W, Pabi SK and Gleiter H. On the mechanism of martensite nucleation. Acta Metallurgica. 1989; 37(1):25-30.

18. Guimarães JRC and Rios PR. Martensite start temperature and the austenite grain-size. Journal Materials Science, 2010, 45(4):1074-1077.

19. Rios PR and Guimarães JRC. Microstructural path analysis of athermal martensite. Scripta Materialia. 2007; 57(12):1105-1108. 
20. Rios PR and Guimarães JRC. Formal Analysis of Isothermal Martensite Spread. Materials Research. 2008; 11(1):103-108.

21. Guimarães JRC and Rios PR. Initial nucleation kinetics of martensite transformation. Journal Materials Science. 2008; 43(15):5206-5210.

22. Guimarães JRC and Rios PR. Unified description of martensite microstructure and kinetics. Journal Materials Science. 2009; 44(4):998-1005.

23. Guimarães JRC and Rios PR (2009), Driving Force and Thermal Activation in Martensite Kinetics. Material and Metallurgical Transactions A. 2009; 40(10):2255-2509.

24. Johnson WA and Mehl RF. Reaction kinetics in processes of nucleation and growth. Transactions AIME. 1939; 135:416-441.

25. Avrami MJ. Kinetics of phase change I general theory. The Journal of Chemical Physics. 1939; 7(12):1103-1112.

26. Kolmogorov NA. The statistics of crystal growth in metals. Isvestiia Academii Nauk SSSR - Seriia Matematicheskaia. 1937; 1:333-359.

27. Gokhale AM, Iswaran CV and DeHoff RT. Use of the stereological counting measurements in testing theories of growth-rates. Metallurgical Transactions A. 1979; 10A(9):1239-1245.
28. DeHoff RT. In: Hansen N, Jensen DJ, Leffers T and Ralph B (editors). Annealing Processes-Recovery, Recrystallization and Grain Growth, Proceedings of the 7th Conference of Ris $\emptyset$ National Laboratory; 1986, Roskilde, Denmark. Roskilde: Ris $\emptyset$ National Laboratory; 1986. p. 3552.

29. Vandermeer RA, Masumura RA and Rath B. Microstructural paths of shape-preserved nucleation and growth transformations. Acta Metallurgica et Materialia. 1991; 39(3):383-389.

30. Rios PR and Villa E. Transformation kinetics for inhomogeneous nucleation. Acta Materialia. 2009; 57(4):1199-1208.

31. Villa E and Rios PR. Transformation kinetics for nucleus clusters. Acta Materialia. 2009; 57(13):3714-3724.

32. Vandermeer RA and Juul Jensen D. Microstructural path and temperature dependence of recrystallization in commercial aluminum. Acta Materialia. 2001; 11(49):2083-2094.

33. Rios PR and Padilha AF. Comment on "Microstructural path and temperature dependence of recrystallization in commercial aluminum". Scripta Materialia. 2003; 48(11):1561-1564.

34. Vandermeer RA. Microstructural descriptors and the effects of nuclei clustering on recrystallization path kinetics. Acta Materialia. 2005; 53(5):1449-1457. 